\title{
Utility and cost-effectiveness of uroflowmetry in a busy pediatric urology practice
}

\author{
Fahad Alyami, MD, FRCSC; Walid Farhat, MD, FRCSC; Victor H. Figueroa, MD; Rodrigo L.P. Romao, MD
}

Division of Pediatric Urology, Department of Surgery, University of Toronto; The Hospital for Sick Children, Toronto, ON

Cite as: Can Urol Assoc J 2014;8(9-10):e615-8. http://dx.doi.org/10.5489/cuaj.2190

Published online September 9, 2014.

\section{Abstract}

Introduction: Uroflowmetry (UF) is frequently employed in daily pediatric urology practice for diagnostic and follow-up purposes. We assess the utility and cost-effectiveness of UF in the management of patients seen at a tertiary care centre.

Methods: We retrospectively reviewed the charts of consecutive patients who had a UF between January 1, 2010 and March 31, 2010. We collected data on demographics, diagnosis, UF parameters and the impact of the UF on management. The impact on management was defined as indication for surgery, introduction of new medications and bladder retraining based on clinical and UF findings.

Results: In total, 524 patients were included in the study. In 63 $(12 \%)$ patients, UF was performed as part of the evaluation at the first clinic appointment. The most common diagnoses were voiding dysfunction (VD) $41 \%$, hypospadias $26 \%$, vesicoureteric reflux (VUR) 16\%, monosymptomatic nocturnal enuresis (NE) 8\%, posterior urethral valves $5 \%$ and meatal stenosis (MS) $4 \%$. In the VD group, UF contributed to a management decision in $25.2 \%$ of patients. In the MS group, surgical intervention was based on symptoms and supported by the UF in $41 \%$ of patients; in the PUV group, $50 \%$ of patients demonstrated high (>20 cc) post-void residual, which aided in management decisions. In contrast, there were virtually no changes in management supported by the UF in the NE, VUR and hypospadias groups. Overall, UF parameters had a direct influence in the management decisions in only 67 $(12.8 \%)$ patients. Nonetheless, a repeat test was ordered for $44.5 \%$ of patients.

Conclusions: In an era of financial restraints and in a busy tertiary pediatric urology practice, judicious use of UF for specific indications may translate into a more cost-effective use of time and resources. As expected, patients with VD were the ones that benefited most from the test, as did patients with symptomatic MS and PUV.

\section{Introduction}

Uroflowmetry (UF) has become a universal investigation that affords urologists a simple, safe, inexpensive and noninvasive way of measuring and recording the urinary flow rate throughout micturition. UF is useful as a screening tool in select patients for more complex urodynamic testing and is an objective method to determine response to treatment of lower urinary tract disorders. ${ }^{1,2}$ The International Continence Society (ICCS) has attempted to standardize certain objective measurements to be recorded during uroflowmetry, including flow curve pattern, maximum flow rate, average flow rate, flow time, voided volume and time to maximum flow. ${ }^{3}$ UF is frequently used in daily pediatric urology (PU) practice for diagnostic and follow-up purposes.

UF has been traditionally used in the voiding dysfunction (VD) population and following hypospadias repairs, but other indications for its use have been described. ${ }^{3}$ For instance, since the diagnosis of VD in children may be challenging to the urologist, UF has been used in conjunction with clinical data, including the history and physical examination, to identify and help treat children with VD. Hypospadias repairs may be also associated with meatal or urethral obstruction with no other apparent symptoms. ${ }^{4}$ Thus, some studies have emphasized the role of urinary flow rate for the functional evaluation in children with hypospadias to detect asymptomatic strictures..$^{5-7}$

In the era of restricted resources in a busy pediatric urology practice, the routine use of UF should be re-evaluated and used selectively. In this study our goal was to evaluate the use of UF in a busy pediatric urology practice and to try to improve and use it for specific patients in a cost-effective fashion.

\section{Methods}

After institutional ethics board approval, we performed a retrospective chart review of all UFs done without electromy- 
Alyami et al.

ography (EMG) consecutively from January 1, 2010 to March 31,2010 . All patients had UF as part of their pediatric urology clinic appointment and available clinical information from the same date was included. Demographics, diagnosis, UF parameters (i.e., voided volume, maximum flow rate, postvoid residual (PVR) and the shape of the curve) and impact of the UF on management were collected. The impact on management was defined as altering intervention, such as the need for surgery, introduction of new medications, bladder retraining or biofeedback and any change in follow-up strategy based solely on clinical and UF findings. We also analyzed the average cost of each UF and the non-contributory studies. There were no exclusion criteria and we included all the performed UF over the 3-month period in the analysis.

\section{Results}

In total, 524 patients (313 male, 211 female) were included in the study. The mean age was $8.2 \pm 3.9$ years. In 63 $(12 \%)$ patients, UF was performed as a part of the initial clinic evaluation. UF was done for the following clinical entities: VD in 215 (41\%), post-hypospadias repair in 136 $(26 \%)$, meatal stenosis (MS) in $21(4 \%)$, nocturnal enuresis in $42(8 \%)$, posterior urethral valves (PUV) in $26(5 \%)$ and vesicourethral reflux (VUR) in 84 (16\%) (Fig. 1).
In the VD group, UF altered the management decision in $25.2 \%$ of patients. In the MS group, surgical intervention was based on symptoms and supported by the UF in $41 \%$ of patients; in the PUV group, $50 \%$ of patients demonstrated high (>20 cc) PVR which helped in management decisions, which included further investigation (like voiding cystogram or cystoscopy), medical (like alpha-blockers) or surgical intervention.

In contrast, there were virtually no changes in management supported by the UF in the NE or VUR group. In fact, only 2 patients $(1.9 \%)$ in the post-hypospadias repair group had surgery indicated based on UF findings coupled with symptoms, despite the finding of a prolonged or flat flow curve in $54 \%$ of patients in this group. Overall, UF parameters had a direct influence in management decisions in only $67(12.8 \%)$ patients. Nonetheless, a repeat test was ordered for 205 out of the remaining 457 patients (44.5\%).

\section{Discussion}

The UF test has gained wide acceptance as the initial screening tool to evaluate voiding function in children because it is simple, non-invasive and inexpensive. ${ }^{8}$ The test is not without its limitations and relies on careful clinical interpretation by a trained urologist. The test should be done in fully

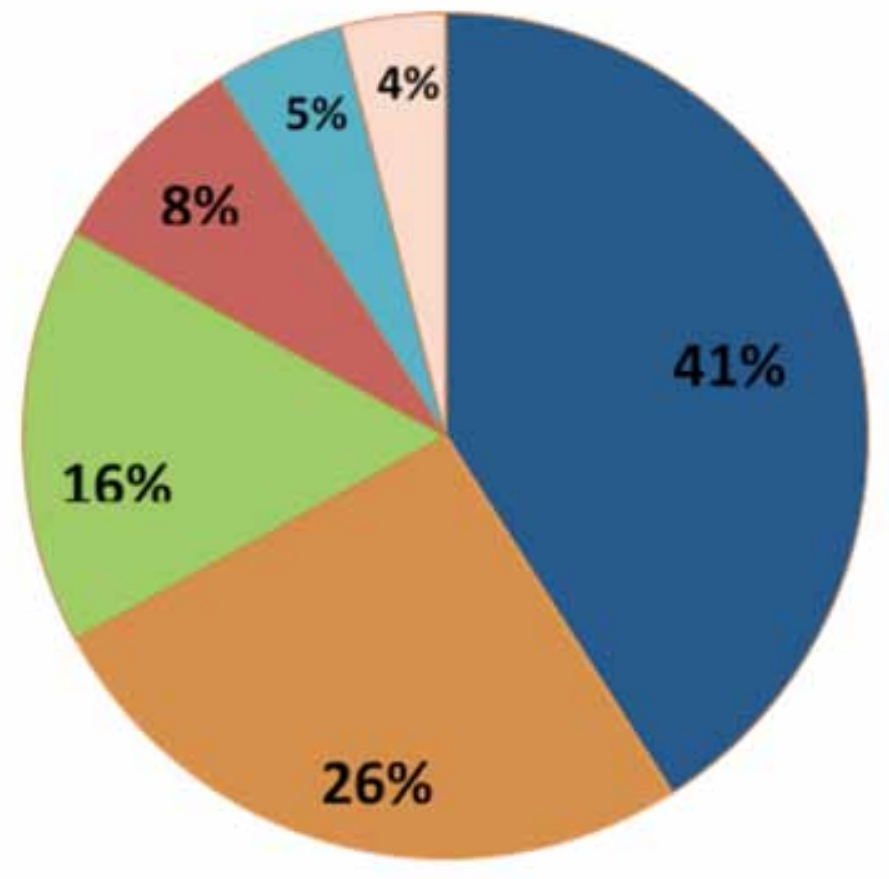

Voiding dysfunction (VD)

Hypospadias

VUR without clinical VD

Monosymptomatic nocturnal enuresis

PUV

Meatal stenois

Fig. 1. Distribution of cases with uroflowmetry. PUV: posterior urethral valves; VUR: vesicoureteric reflux; VD: voiding dysfunction. 
toilet-trained children with adequate voided volume $(>50 \%$ of the expected bladder capacity) to generate adequate curve and improve the accuracy and the reliability of the interpreted test. Clinically useful UF parameters include flow rate (maximum and average), the flow rate curve shape, voided volume and the PVR. Currently, there is a lack of accepted standardized normal values for the measured UF parameters in children as most publications in the pediatric population contain wide age ranges and combine both genders. ${ }^{9-12}$ There is also little evidence with regards to the usefulness and effectiveness of UF for different conditions in children. ${ }^{13}$

UF has been used commonly in patients with VD and postoperative hypospadias and our data support these indications. Diagnosis of VD in children can be a challenge to pediatric urologists. Urodynamic examination is generally one of most reliable tests, but also the most invasive tool to detect pelvic floor disorders and detrusor overactivity. UF is a non-invasive version of the urodynamics studies, which can be added to the constellation of clinical data to identify and treat VD in children with the least possible trauma to the child. Schewe and colleagues found that full urodynamic testing is of limited value in the diagnosis of VD in children, and may lead to treatment decisions in only $7 \%$ of patients. ${ }^{14}$

Evaluating long-term results of hypospadias repairs is important because the operation is usually performed early in life and the long-term results are sometimes unknown. UF is traditionally used to evaluate the functional results following hypospadias repair in addition to the history and physical exam to diagnose any obstruction related to the initial surgery. No study has linked UF findings to symptoms, if any, although a number of authors commented that patients with peak flows $<5$ th percentile were not further investigated for the lack of symptoms. Multiple studies showed that plateaushaped curve and/or low peak flow rates after hypospadias repair does not indicate urethral obstruction. ${ }^{5,7,15}$ However, until long-term follow-up studies clarify the significance of abnormal UF, the significance of these studies remain uncertain. In MS and PUV patients, the UF is a good adjunct test at the time of diagnosis. The long-term follow-up for these patients is important, but the need for routine UF is unknown. The need for routine UF post-hypospadias repair, MS and PUV in asymptomatic children during follow-up is questionable, but may aid in decision-making for symptomatic patients.

In the current study we reviewed the use of UF over a 3-month period. UF contributed to management decisions in symptomatic VD, MS and PUV patients. In contrast, there were virtually no changes in management supported by the UF in the NE, VUR and asymptomatic hypospadias patients. Although UF is routinely done for hypospadias patients during their postoperative follow-ups, only 2 patients $(1.9 \%)$ in the post-hypospadias repair group had surgery indicated based on UF findings coupled with symptoms. Overall, UF parameters had a direct influence in the management decisions in only $67(12.8 \%)$ patients. Nonetheless, a repeat test was ordered for 205 out of the remaining 457 patients (44.5\%).

At our institution, we see about 190 patients per week. During the 3-month period we did a total of 524 UF studies, which meant we did about 11 UFs per clinic. The approximate cost of a single UF study at our center is about $\$ 36.45$ CAD per study. The time needed to complete each study (given that patient is ready [full bladder] is about 5 minutes. The time commitment of the nursing staff is an average of 55 minutes per week. The cost of noncontributory studies during the 3-month study period was $\$ 16657.65$ CAD, keeping in mind that there are additional indirect costs, such as prolonged waiting time and the need for multiple attempts.

Our study has several limitations. Firstly, the retrospective cross-sectional design had a limited ability to portray the actual indication for the UF. Our conclusions were drawn based on a snapshot that may not have taken into account more complex issues involved in the decision to perform the test; moreover, the time of the UF in the course of the management was variable. Secondly, there was significant variability in age, gender and diagnosis and this may have skewed the data in an unpredictable way. Nonetheless, we found an overutilization of UF, which is important to take into consideration, especially in terms of planning for judicious use of resources at a tertiary care centre.

\section{Conclusion}

In an era of financial constraints and in the context of a busy tertiary pediatric urology clinic, the judicious use of UF may translate into a more cost-effective use of time and resources. As expected, patients with VD were the ones who benefited most from the test, as did patients with symptomatic MS and PUV. Patients seen for NE and VUR may not need a UF at all. Despite being frequently abnormal, UF in asymptomatic patients post-hypospadias repair had a limited impact on management and should be done for symptomatic patients only. We believe that the non-invasive nature of the UF made it a very popular test to identify subclinical bladder or uretheral issues; it is very possible that patients with normal UF were discharged based on a normal test. We currently have changed our practice and adopted a very selective UF testing and we hope in future studies to show that this new criteria for UF testing has a higher yield to identify patients with emptying problems.

Competing interests: Dr. Alyami, Dr. Farhat, Dr. Figueroa and Dr. Romao all declare no competing financial or personal interests. 
Alyami et al.

This paper has been peer-reviewed.

\section{References}

1. Hialmas K, Hoebeke PB, de Paepe H. Lower urinary tract dysfunction and urodynamics in children. Eur Urol 2000;38:655. http://dx.doi.org/10.1159/000020351

2. Wein AJ, Barrett DM. Physiology of micturition and urodynamics. In: Kelalis P.P., ed. Clinical Pediatric Urology. Philadelphia, PA: WB Saunders; 1992:187-217.

3. Abrams $P$, Khoury $S$, Wein A. Incontinence. Proceedings of the First International Consultation on Incontinence 1998. Plymouth, United Kingdom: Health Publications Ltd; 1999:933-69.

4. Schertz HC, Kaplan GW, Packer MG, et al. Post-hypospadias repair urethral strictures: A review of 30 cases. J Urol 1988; 140:1253-5.

5. Marte A, Di lorio $G$, De Pasquale $M$, et al. Functional evaluation of TIPU repairs of midshaft-proximal hypospadias using uroflowmetry. BJU Int 2001;87:540-3. http://dx.doi.org/10.1046/j.1464410X.2001.00090.x

6. Garibay JT, Raid C, Gonzales R. Functional evaluation of the results of hypospadias surgery with uroflowmetry J Urol 1995;154:835-6. http://dx.doi.org/10.1016/S0022-5347(01)67178-7

7. Hammouda HM, El-Ghoneimi A, Bagli DJ, et al. Tubularized incised plate repair: Functional outcome after intermediate follow-up. J Urol 2003;169:331-3. http://dx.doi.org/10.1016/S0022-5347(05)64120-1

8. Neveus $\mathrm{T}$, von Gontard A, Hoebeke P, et al. The standardization of terminology of lower urinary tract function in children and adolescents: Report from the Standardisation Committee of the International Children's Continence Society. J Urol 2006;176:314-24. http://dx.doi.org/10.1016/S0022-5347(06)00305-3
9. Haylen BT, Ashby D, Sutherst JR, et al. Maximum and average urine flow rates in normal male and female populations— the Liverpool nomograms. Br J Urol 1989;64:30. http://dx.doi.org/10.1111/i.1464410X.1989.tb05518.x

10. Szabo L, Fegyverneki S. Maximum and average urine flow rates in normal children-the Miskolc nomograms. Br J Urol 1995;76:16. http://dx.doi.org/10.1111/i.1464-410X.1995.tb07824.x

11. Kajbafzadeh AM, Yazdi CA, Rouhi 0 , et al. Uroflowmetry nomogram in Iranian children aged 7 to 14 years. BMC Urol 2005;5:3. http://dx.doi.org/10.1186/1471-2490-5-3

12. Pernkopf D, Plas E, Lang T, et al. Uroflow nomogram for male adolescents. J Urol 2005;174:1436. http://dx.doi.org/10.1097/01.ju.0000173694.86127.ae

13. Kumar V, Dhabalia JV, Nelivigi GG, et al. Age, gender, and voided volume dependency of peak urinary flow rate and uroflowmetry nomogram in the Indian population. Indian I Urol 2009;25:461. hittp:// dx.doi.org/10.4103/0970-1591.57912

14. Schewe J, Brands FH, Pannek J. Voiding dysfunction in children: Role of urodynamic studies. Urol Int 2002;69:297-301. http://dx.doi.org/10.1159/000066129

15. Jayanthi VR, McLorie GA, Khoury AE, et al. Functional characteristics of the reconstructed neourethra after island flap urethroplasty. J Urol 1995;153:1657. http://dx.doi.org/10.1016/S0022$5347(01) 67496-2$

Correspondence: Dr. Fahad Alyami, Division of Pediatric Urology, Department of Surgery, University of Toronto, Toronto, ON; fahad.alyami@sickkids.co 